\title{
Molecular characterization of sweet cherry genetic resources in Giresun, Turkey
}

\author{
Taki DEMIR $^{1 *}$, Leyla DeMIRSOY ${ }^{2}$, Hüsnü DeMIRSOY ${ }^{2}$, YıIdız AKA KAÇAR $^{3}$, Muharrem YILMAZ ${ }^{3}$, Idris MACIT ${ }^{4}$
}

\author{
${ }^{1}$ Sakarya Univ., Vocat. Sch. \\ Geyve, 54700, Geyve, \\ Sakarya, Turkey, \\ tdemir@sakarya.edu.tr \\ 2 Ondokuz Mayıs Univ., Fac. \\ Agric., Dep. Hortic., Samsun, \\ Turkey \\ ${ }^{3}$ Cukurova Univ., Fac. Agric., \\ Dep. Hortic., Adana 01330, \\ Turkey \\ ${ }^{4}$ Karadeniz Agric. Res. Inst., \\ Samsun, Turkey
}

* Correspondence and reprints

Received 8 April 2009
Accepted 16 June 2009

Fruits, 2011, vol. 66, p. 53-62 (C) 2011 Cirad/EDP Sciences All rights reserved DOI: 10.1051/fruits/2010041 www.fruits-journal.org

RESUMEN ESPAÑOL, p. 62

\section{Molecular characterization of sweet cherry genetic resources in Giresun, Turkey.}

Abstract - Introduction. Turkey potentially has a very rich source of sweet (Prunus avium) and sour ( $P$. cerasus) cherries. P. avium is apparently native to some parts of Northern Turkey, where Giresun is located. Identification of the sweet cherry cultivars produced in Turkey will help in choosing appropriate cultivars and aid in the preservation of natural resources required for breeding studies. The most conventional method of cultivar identification is based on the assessment of morphological characteristics. However, this method is insufficient to distinguish closely related cultivars. The aims of our study were to determine the molecular profile of sweet cherry accessions grown in Giresun, Turkey, and to determine their genetic relationships. Materials and methods. In our study, we identified 44 sweet cherry accessions grown in Giresun by using genetic markers (SSR, Simple Sequence Repeat), and we determined the genetic relationships among the sweet cherry genotypes. For DNA isolation, we collected young leaves sampled on a single plant per accession, then amplification of microsatellite loci was performed. In total, ten SSR primer pairs, previously isolated from peach and sweet cherry, were used. Genetic similarity values were calculated. A cluster analysis was performed to generate a dendrogram. Results and discussion. Of the ten primers tested, six primer pairs did not result in suitable amplification products with the 44 accessions studied. The remaining four polymorphic SSR primer pairs produced 33 alleles with an average of 8.25 putative alleles per locus, ranging from 7 to 11 . Depending on the accessions, similarity ratios ranged from 0.32 to 0.98 , with a mean value of 0.64 . In conclusion, the results obtained demonstrate a high level of polymorphism among sweet cherry genotypes from a single province in Turkey.

Turkey / Prunus avium / genetic resources / identification / microsatellites / genetic markers / genetic distance

\section{Caractérisation moléculaire des ressources génétiques du cerisier dans la province de Giresun en Turquie.}

Résumé - Introduction. La Turquie serait potentiellement une source très riche de cerisiers doux (Prunus avium) et aigres (Prunus cerasus). L'espèce $P$. avium serait indigène de certaines régions du nord de la Turquie, dont la province de Giresun. L'identification des cultivars de cerisiers turcs devrait aider à faire des choix et contribuer à la conservation des ressources naturelles nécessaires à des travaux d'amélioration. La méthode la plus conventionnelle pour l'identification de cultivars est basée sur l'évaluation des caractéristiques morphologiques. Cependant, cette méthode est insuffisante pour distinguer les cultivars étroitement liés. Les objectifs de notre étude ont été de déterminer le profil moléculaire d'accessions de cerisiers produits dans la province de Giresun en Turquie et de déterminer leurs distances génétiques. Matériel et méthodes. Notre étude a permis d'identifier 44 accessions de cerisiers grâce à l'utilisation de marqueurs génétiques et de déterminer leurs distances génétiques. Nous avons isolé l'ADN de jeunes feuilles prélevées sur un plant par accession, et nous avons effectué l'amplification des loci de microsatellites. Au total, dix paires d'amorce de SSR, précédemment isolées de pêcher et de cerisier, ont été employées. Des indices de similitude ont été calculés. Une analyse typologique a été utilisée pour tracer un dendrogramme. Résultats et discussion. Des dix amorces examinées, six n'ont pas permis d'obtenir de produits d'amplification à partir des 44 accessions étudiées. Les quatre paires d'amorce polymorphes restantes ont produit 33 allèles avec une moyenne de 8.25 allèles putatifs par locus. Selon les accessions, les indices de similitude ont varié de 0.32 à 0.98 , avec une valeur moyenne de 0.64. En conclusion, les résultats obtenus révèlent un niveau élevé de polymorphisme parmi les génotypes de cerisier étudiés dans une seule province de la Turquie.

Turquie / Prunus avium / ressource génétique / identification / microsatellite / marqueur génétique / distance génétique 


\section{Introduction}

Anatolia is the motherland of sweet cherries [1]. It has been stated that Turkey potentially has a very rich source of sweet (Prunus avium) and sour (P. cerasus) cherries [1-6]. The sweet cherry is apparently native to some parts of Northern Turkey [7], where Giresun is located. In Turkey, many cherry genotypes and local cultivars could contribute to the domesticated sweet cherry. Unfortunately, some of these genetic resources have become extinct, but those that remain may be genetic resources for breeding studies striving to improve traits such as resistance, developing new cultivars, improving fruit quality and extending the cherry production season.

There are about 1,500 cultivars of sweet cherry in the world. The names of the cherry cultivars are much more complicated than those of any other cultivars of fruit. Some cultivars are highly similar. Although sometimes the same cultivars are called different names, it is also the case that sometimes different cultivars are called by the same name $[1,2]$. For this reason, cultivars and types of sweet cherry show a great variability in Anatolia. Identification of the sweet cherry cultivars produced in Turkey will help in choosing appropriate cultivars and aid in the preservation of natural resources required for breeding studies.

The most conventional method of cultivar identification is based on the assessment of morphological characteristics. However, this method is insufficient to distinguish closely related cultivars. Furthermore, variation in biochemical characteristics (e.g., isozymes) can be useful for the determination of cultivar identity [8, 9]. However, it has been shown that the isozyme polymorphism level is low in sweet cherries [10]. In recent years, new methods that can detect differences at the genomic level have been developed. For example, RAPD (Random Amplified Polymorphic DNA) has been used for identifying and determining the relationships among cultivars [11]. Yang and Schmitt distinguished mutant cherries and their parents using RAPD primers [12]. Corona et al. [13] and Gerlach and Stösser [14] genetically identified 15 and 18 sweet cherry cultivars, respectively, using RAPD fingerprints. RAPD and SSR methods have also been successfully used in Turkey in order to identify sweet cherry and sour cherry cultivars [1, 4-6, 15]. Additionally, DNA fingerprinting methods including SSR [16-18] and AFLP [10, 16, 18, 19] have been used for cultivar identification in sweet cherry.

The aims of our study were to determine with SSR markers the molecular profile of 44 sweet cherry accessions grown in Giresun, Turkey, and to determine their genetic relationships.

\section{Materials and methods}

\subsection{Plant materials}

The sweet cherry genotypes that we examined in our studies have been grafted onto different cherry rootstocks (Gisela5, Gisela6, and Mazzard) and preserved in the orchard of the Karadeniz Agricultural Research Institute (Samsun, Turkey). We used 44 sweet cherry accessions as plant material (table I). They were collected from Giresun (Turkey), one of the centers of sweet cherry cultivation (figure 1).

\subsection{DNA isolation}

For DNA isolation, young leaves were collected from a single plant for each accession; they were frozen in liquid nitrogen and stored at $-80{ }^{\circ} \mathrm{C}$. Genomic DNA was isolated from the leaves using the DNeasy Plant Mini Kit (QIAGEN, GmbH, Germany) according to the manufacturer's instructions. DNA concentrations were measured using a spectrophotometer. DNA was diluted in water to a final concentration of $50 \mathrm{ng} \cdot \mathrm{LL}^{-1}$ and stored at $-20{ }^{\circ} \mathrm{C}$.

\subsection{PCR amplification of microsatellite loci}

DNA amplification was performed in a volume of $25 \mu \mathrm{L}$, containing $20 \mathrm{ng}$ template DNA, $12.5 \mu \mathrm{L}$ PCR Master Mix 2× (50 mM 


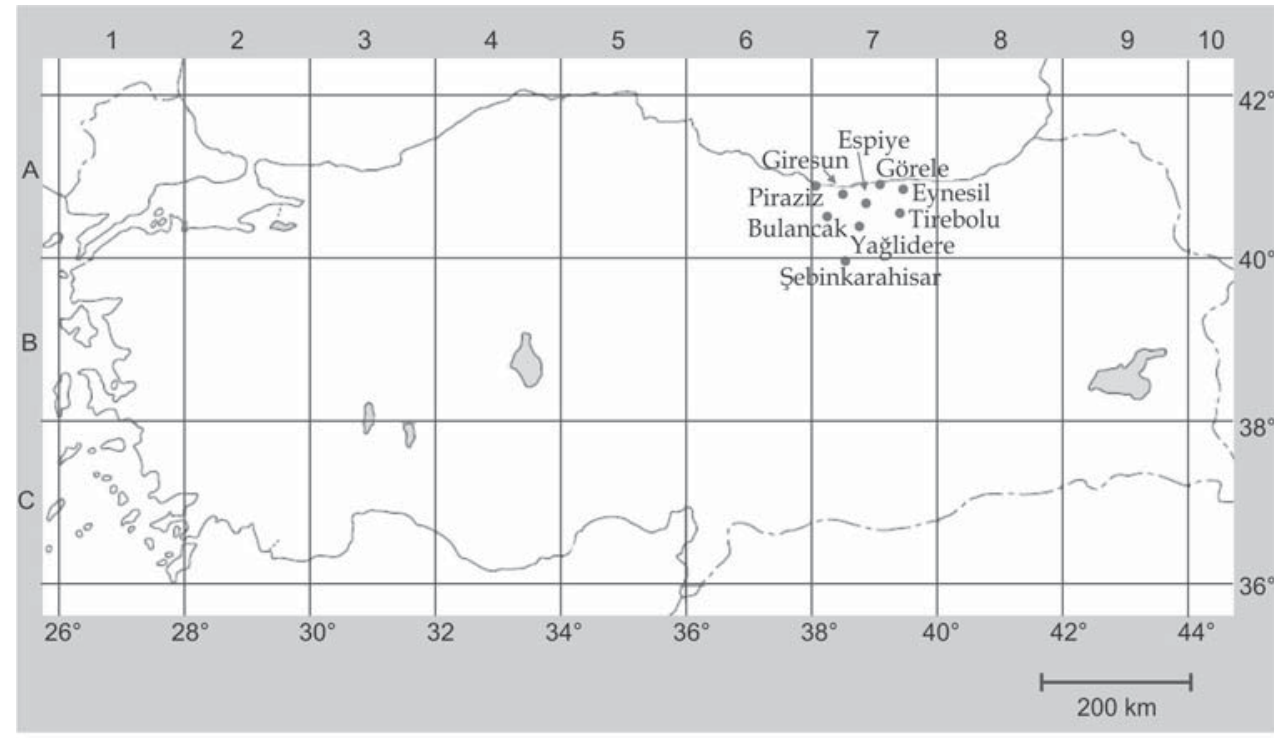

Figure 1.

General distribution of 44 collected sweet cherry accessions grown in Giresun, Turkey.
Tris- $\mathrm{HCl} \mathrm{pH} 9.0,50 \mathrm{mM} \mathrm{NaCl}, 3 \mathrm{mM} \mathrm{MgCl}_{2}$, $400 \mu \mathrm{M}$ dATP, dGTP, dCTP, dTTP, 50 unit $\cdot \mathrm{mL}^{-1}$ Taq DNA polymerase) (Promega M7505, Promega Corporation, Madison, WI, USA), $2.5 \mathrm{pmol}$ forward $(2.0 \mu \mathrm{L})$ and $2.5 \mathrm{pmol}$ reverse $(2.0 \mu \mathrm{L}) \mathrm{SSR}$ primer pairs, $2.5 \mathrm{mM} \mathrm{MgCl} 2,0.6$ unit $\cdot \mathrm{mL}^{-1}$ Taq DNA Polymerase (Promega M1665, Promega Corporation, Madison, WI, USA), and $3.375 \mu \mathrm{L} \mathrm{ddH}_{2} \mathrm{O}$ (nuclease-free). The amplifications were performed in an Eppendorf Mastercycler Gradient programed for $3 \mathrm{~min}$ at $94^{\circ} \mathrm{C}$ for initial denaturation, then 35 cycles of $30 \mathrm{sec}$ at $94^{\circ} \mathrm{C}$ for denaturation, $30 \mathrm{sec}$ at $50-58{ }^{\circ} \mathrm{C}$ for annealing (annealing temperature was determined for each primer pair specifically) and $30 \mathrm{sec}$ at $72{ }^{\circ} \mathrm{C}$ for polymerization, followed by a final polymerization step of $7 \mathrm{~min}$ at $72{ }^{\circ} \mathrm{C}$.

In total, ten SSR primer pairs, previously isolated from peach and sweet cherry, were used for DNA amplification (table II).

The amplified products were separated in a $6.0 \%$ polyacrylamide gel in a Sequi-Gen ${ }^{\circledR}$ Sequencer (BIO RAD Laboratories Inc., USA), run at $80 \mathrm{~W}$ for $2.5 \mathrm{~h}$ and stained with the Silver Sequence Staining System (Promega, Madison, WI, USA). The molecular sizes of the amplification products were estimated using a 10-bp ladder (Gibco BRL, Rockville, MD, USA).

\subsection{Data analysis}

For each primer pair, SSR fragments were scored as present (1) or absent (0). Genetic similarity values were calculated by Dice coefficients [22]. Unweighted Pair-Group Method Analysis (UPGMA) was performed to generate a dendrogram with NTSYS-pc software, version $2.02 \mathrm{i}$ (Exeter Software, Stauket, New York, NY, USA).

\section{Results and discussion}

In our study, of the ten primers tested, six primer pairs (PS08E08, PceGA77, PceGA34, PS12A02, PceGA25 and PMS30) did not result in suitable amplification products with the accessions studied. Four polymorphic SSR primer pairs produced 33 alleles with an average of 8.25 putative alleles per locus, ranging from 7 (Pchpgms3) to 11 (PMS2) (table III). The 8.25 alleles per locus obtained is higher than that reported by Kaçar [2] (3.4 alleles per locus) and Öz et al. [6] (5.25 alleles per locus) and lower than that reported by Kaçar et al. [5] (12.6 alleles per locus). Kaçar reported that, in sweet cherry, the number of alleles per locus ranges according to the number of genotypes [2]. A higher number of genotypes 


\section{T. Demir et al.}

Table I.

Location of sweet cherry accessions from Giresun, Turkey, which were studied for a molecular characterization.

\begin{tabular}{|c|c|c|c|c|c|}
\hline \multirow[t]{2}{*}{ Accession name } & \multirow[t]{2}{*}{ Accession code } & \multirow[t]{2}{*}{ Location } & \multirow{2}{*}{$\begin{array}{c}\text { Altitude } \\
\text { (m) }\end{array}$} & \multicolumn{2}{|c|}{ Directions } \\
\hline & & & & Lat. (N) & Long. (E) \\
\hline Tirebolu1 & $\mathrm{T} 1$ & Harşit Valley & 106 & $41^{\circ} 0^{\prime} 11^{\prime \prime}$ & $38^{\circ} 30^{\prime} 55^{\prime \prime}$ \\
\hline Tirebolu2 & $\mathrm{T} 2$ & & 106 & $41^{\circ} 0^{\prime} 11^{\prime \prime}$ & $38^{\circ} 30^{\prime} 55^{\prime \prime}$ \\
\hline Tirebolu3 & T3 & Orta Cami Karakaya area & 280 & $40^{\circ} 35^{\prime} 3^{\prime \prime}$ & $38^{\circ} 32^{\prime} 52^{\prime \prime}$ \\
\hline Tirebolu4 & $\mathrm{T} 4$ & & 280 & $40^{\circ} 35^{\prime} 3^{\prime \prime}$ & $38^{\circ} 32^{\prime} 52^{\prime \prime}$ \\
\hline Tirebolu5 & T5 & & 273 & $40^{\circ} 34^{\prime} 55^{\prime \prime}$ & $38^{\circ} 32^{\prime} 52^{\prime \prime}$ \\
\hline Piraziz1 & $\mathrm{P} 1$ & Kökçeali village & 608 & $40^{\circ} 31^{\prime} 56^{\prime \prime}$ & $38^{\circ} 3^{\prime} 20^{\prime \prime}$ \\
\hline Piraziz2 & P2 & & 594 & $40^{\circ} 31^{\prime} 51^{\prime \prime}$ & $38^{\circ} 3^{\prime} 22^{\prime \prime}$ \\
\hline Piraziz3 & P3 & Çayırköy village & 429 & $40^{\circ} 31^{\prime} 24^{\prime \prime}$ & $38^{\circ} 2^{\prime} 55^{\prime \prime}$ \\
\hline Piraziz4 & P4 & & 452 & $40^{\circ} 31^{\prime} 25^{\prime \prime}$ & $38^{\circ} 2^{\prime} 51^{\prime \prime}$ \\
\hline Piraziz5 & P5 & & 604 & $40^{\circ} 31^{\prime} 17^{\prime \prime}$ & $38^{\circ} 2^{\prime} 38^{\prime \prime}$ \\
\hline Yağlıdere1 & $\mathrm{Y}_{1}$ & Yeşilçınar Molla District & 741 & $40^{\circ} 23^{\prime} 56^{\prime \prime}$ & $38^{\circ} 21^{\prime} 48^{\prime \prime}$ \\
\hline Yağlıdere2 & Y2 & & 762 & $40^{\circ} 23^{\prime} 54^{\prime \prime}$ & $38^{\circ} 21^{\prime} 49^{\prime \prime}$ \\
\hline Yağlıdere3 & Y3 & & 808 & $40^{\circ} 23^{\prime} 53^{\prime \prime}$ & $38^{\circ} 21^{\prime} 58^{\prime \prime}$ \\
\hline Yağlıdere4 & Y4 & & 827 & $40^{\circ} 23^{\prime} 50^{\prime \prime}$ & $38^{\circ} 22^{\prime} 8^{\prime \prime}$ \\
\hline Yağlıdere5 & Y5 & & 900 & $40^{\circ} 23^{\prime} 52^{\prime \prime}$ & $38^{\circ} 22^{\prime} 6^{\prime \prime}$ \\
\hline Yağlıdere6 & Y6 & & 908 & $40^{\circ} 23^{\prime} 53^{\prime \prime}$ & $38^{\circ} 22^{\prime} 5^{\prime \prime}$ \\
\hline Espiye3 & ES3 & Ağalık District & - & $40^{\circ} 32^{\prime} 16^{\prime \prime}$ & $38^{\circ} 25^{\prime} 33^{\prime \prime}$ \\
\hline Cent. Giresun1 & GM1 & Erikliman village & 22 & $40^{\circ} 33^{\prime} 27^{\prime \prime}$ & $38^{\circ} 10^{\prime} 55^{\prime \prime}$ \\
\hline Cent. Giresun2 & GM2 & & 42 & $40^{\circ} 33^{\prime} 27^{\prime \prime}$ & $38^{\circ} 11^{\prime} 1^{\prime \prime}$ \\
\hline Cent. Giresun3 & GM3 & & 32 & $40^{\circ} 33^{\prime} 28^{\prime \prime}$ & $38^{\circ} 11^{\prime} 1^{\prime \prime}$ \\
\hline Cent. Giresun4 & GM4 & & 31 & $40^{\circ} 33^{\prime} 28^{\prime \prime}$ & $38^{\circ} 11^{\prime} 2^{\prime \prime}$ \\
\hline Cent. Giresun5 & GM5 & Gürköy village & 400 & - & - \\
\hline Cent. Giresun6 & GM6 & & 400 & - & - \\
\hline Cent. Giresun7 & GM7 & & 400 & $40^{\circ} 30^{\prime} 33^{\prime \prime}$ & $38^{\circ} 15^{\prime} 31^{\prime \prime}$ \\
\hline Cent. Giresun8 & GM8 & & - & $40^{\circ} 32^{\prime} 24^{\prime \prime}$ & $38^{\circ} 14^{\prime} 42^{\prime \prime}$ \\
\hline Cent. Giresun9 & GM9 & Elma Tepesi & 946 & $40^{\circ} 28^{\prime} 22^{\prime \prime}$ & $38^{\circ} 13^{\prime} 43^{\prime \prime}$ \\
\hline Cent. Giresun10 & GM10 & & - & $40^{\circ} 32^{\prime} 24^{\prime \prime}$ & $38^{\circ} 14^{\prime} 42^{\prime \prime}$ \\
\hline Cent. Giresun11 & GM11 & & - & - & - \\
\hline Cent. Giresun12 & GM12 & & 510 & $40^{\circ} 31^{\prime} 23^{\prime \prime}$ & $38^{\circ} 13^{\prime} 36^{\prime \prime}$ \\
\hline Bulancak1 & B1 & Pazarsuyu village & 93 & $40^{\circ} 33^{\prime} 32^{\prime \prime}$ & $38^{\circ} 6^{\prime} 48^{\prime \prime}$ \\
\hline Bulancak2 & B2 & & 80 & $40^{\circ} 33^{\prime} 27^{\prime \prime}$ & $38^{\circ} 6^{\prime} 37^{\prime \prime}$ \\
\hline Görele1 & G1 & Kırıkıı & 320 & $40^{\circ} 35^{\prime} 11^{\prime \prime}$ & $39^{\circ} 2^{\prime} 44^{\prime \prime}$ \\
\hline Görele2 & G2 & & - & - & - \\
\hline Şebinkarahisar1 & $\mathrm{S} 1$ & Tamzara location & 1380 & $40^{\circ} 12^{\prime} 42^{\prime \prime}$ & $38^{\circ} 16^{\prime} 13^{\prime \prime}$ \\
\hline Şebinkarahisar2 & $\mathrm{S} 2$ & & 1373 & $40^{\circ} 12^{\prime} 51^{\prime \prime}$ & $38^{\circ} 16^{\prime} 0^{\prime \prime}$ \\
\hline Şebinkarahisar3 & S3 & Hacıömer village & - & - & - \\
\hline Eynesil1 & E1 & Kekiktepe & 74 & - & - \\
\hline Eynesil2 & E2 & & 64 & - & - \\
\hline Eynesil3 & E3 & & 174 & $41^{\circ} 1^{\prime} 30^{\prime \prime}$ & $39^{\circ} 3^{\prime} 31^{\prime \prime}$ \\
\hline Eynesil4 & E4 & & 246 & $41^{\circ} 1^{\prime} 11^{\prime \prime}$ & $39^{\circ} 3^{\prime} 36^{\prime \prime}$ \\
\hline Eynesil5 & E5 & & 284 & $41^{\circ} 1^{\prime} 8^{\prime \prime}$ & $39^{\circ} 4^{\prime} 4^{\prime \prime}$ \\
\hline Eynesil6 & E6 & & 314 & $41^{\circ} 4^{\prime} 49^{\prime \prime}$ & $39^{\circ} 3^{\prime} 43^{\prime \prime}$ \\
\hline Eynesil7 & E7 & & 324 & $41^{\circ} 1^{\prime} 3^{\prime \prime}$ & $39^{\circ} 3^{\prime} 44^{\prime \prime}$ \\
\hline Eynesil8 & E8 & Kemerli village & 481 & $41^{\circ} 0^{\prime} 2^{\prime \prime}$ & $39^{\circ} 4^{\prime} 10^{\prime \prime}$ \\
\hline
\end{tabular}




\begin{tabular}{|c|c|c|c|c|}
\hline Primer pair & Orientation & Sequence 5'...3' & Reference & Source \\
\hline \multirow[t]{2}{*}{ Pchpgms3 } & Forward & ACGCTATGTCCGTACACTCTCCATG & [20] & Peach \\
\hline & Reverse & СААССТGTGАТTGСТССТАТTАААС & & \\
\hline \multirow[t]{2}{*}{ PMS2 } & Forward & САCTGTCTCCCAGGTTAAACTC & [21] & Sweet Cherry \\
\hline & Reverse & CCTGAGCTITTGACACATGC & & \\
\hline \multirow[t]{2}{*}{ PMS3 } & Forward & TGGACTTCACTCATTTCAGAGA & [21] & Sweet Cherry \\
\hline & Reverse & ACTGCAGAGAATTTCACAACCA & & \\
\hline \multirow[t]{2}{*}{ PMS67 } & Forward & AGTCGCTCACAGTCAGTTTCTC & [21] & Sweet Cherry \\
\hline & Reverse & ТТААСТТААССССТСТСССТСС & & \\
\hline \multirow[t]{2}{*}{$\mathrm{PS} 8 \mathrm{E} 08^{1}$} & Forward & СССААТGAАСАAСТGСАТ & {$[20]$} & Sweet Cherry \\
\hline & Reverse & CATATCAATCACTGGGATG & & \\
\hline \multirow[t]{2}{*}{ PceGA77 1} & Forward & CCTTACCACTGGCATCATCA & [21] & Sour Cherry \\
\hline & Reverse & CAGCTGAGCAGGCAACAAAA & & \\
\hline \multirow[t]{2}{*}{ PceGA34 ${ }^{1}$} & Forward & GAACATGTGGTGTGCTGGTT & {$[17]$} & Sour Cherry \\
\hline & Reverse & TCCACTAGGAGGTGCAAATG & & \\
\hline \multirow[t]{2}{*}{$\mathrm{PS} 12 \mathrm{~A} 02^{1}$} & Forward & GCCACCAATGGTTCTTCC & {$[17]$} & Sweet Cherry \\
\hline & Reverse & AGCACCAGATGCACCTGA & & \\
\hline \multirow[t]{2}{*}{ PceGA25 ${ }^{1}$} & Forward & GCAATTCGAGCTGTATTTCAGATG & [21] & Sour Cherry \\
\hline & Reverse & CAGTTGGCGGCTATCATGCTTAC & & \\
\hline \multirow[t]{2}{*}{$\mathrm{PMS} 0^{1}$} & Forward & CTGTCGAAAGTTTGCCTATGC & [18] & Sweet Cherry \\
\hline & Reverse & ATGAATGCTGTGTACATGAGG & & \\
\hline
\end{tabular}

gave a higher number of alleles per locus. One of the reasons for a higher number of alleles per locus (8.25) can be related to a higher number of genotypes. Because Kaçar et al. used a high number of genotypes (81 sour cherry cultivars, open-pollinated types and accessions) for SSR analysis [5], their results (12.6 alleles per locus) were higher than our results (8.25 alleles per locus). On the other hand, polyploid individuals have a higher number of alleles per locus than diploid individuals. So, sweet cherry (Prunus avium) includes diploid individuals, but sour cherry is tetraploid. Therefore, our results are in agreement with the results of other authors [1, 4-6, 17, 20, 21]. Obtaining a higher number of alleles per locus verified that there is a higher variability among the investigated sweet cherry genotypes in our research.

The PMS2 primer pair, isolated from sweet cherry, produced 11 alleles. PMS2 is the most informative primer pair used in our study (table III). The number of alleles that we obtained with this primer pair was higher than the numbers reported by Cantini et al. [21], Kaçar [2] and Kaçar et al. [4].

The PMS3 primer pair produced eight informative putative alleles. This number is higher than that reported by Kaçar et al. (4 alleles) [4] and lower than those reported by Cantini et al. (16 alleles) [21], Kaçar (11 alleles) [2] and Kaçar et al. (17 alleles) [5].

Seven putative alleles were obtained from primer pair PMS67. This allele number is higher than those reported by Kaçar [2] and Kaçar et al. (3 alleles) [4] and lower than that reported by Cantini et al. (13 alleles) [21].

The primer pair Pchpgms3, isolated from peach, produced seven putative alleles, one of which is monomorphic. This primer was informative for sweet cherry. The number of alleles that we obtained is lower than that of Downey and Iezzoni (15 alleles) [17] and higher than the reports of Sosinski et al. (1 allele) [20], Cantini et al. [21], Kaçar [2], Kaçar et al. [5], Öz et al. (5 alleles) [6] and 


\section{T. Demir et al.}

Figure 2.

Unweighted Pair-Group Method Analysis (UPGMA) dendrogram of 44 sweet cherry accessions from Giresum (Turkey) constructed based on 33 SSR markers.

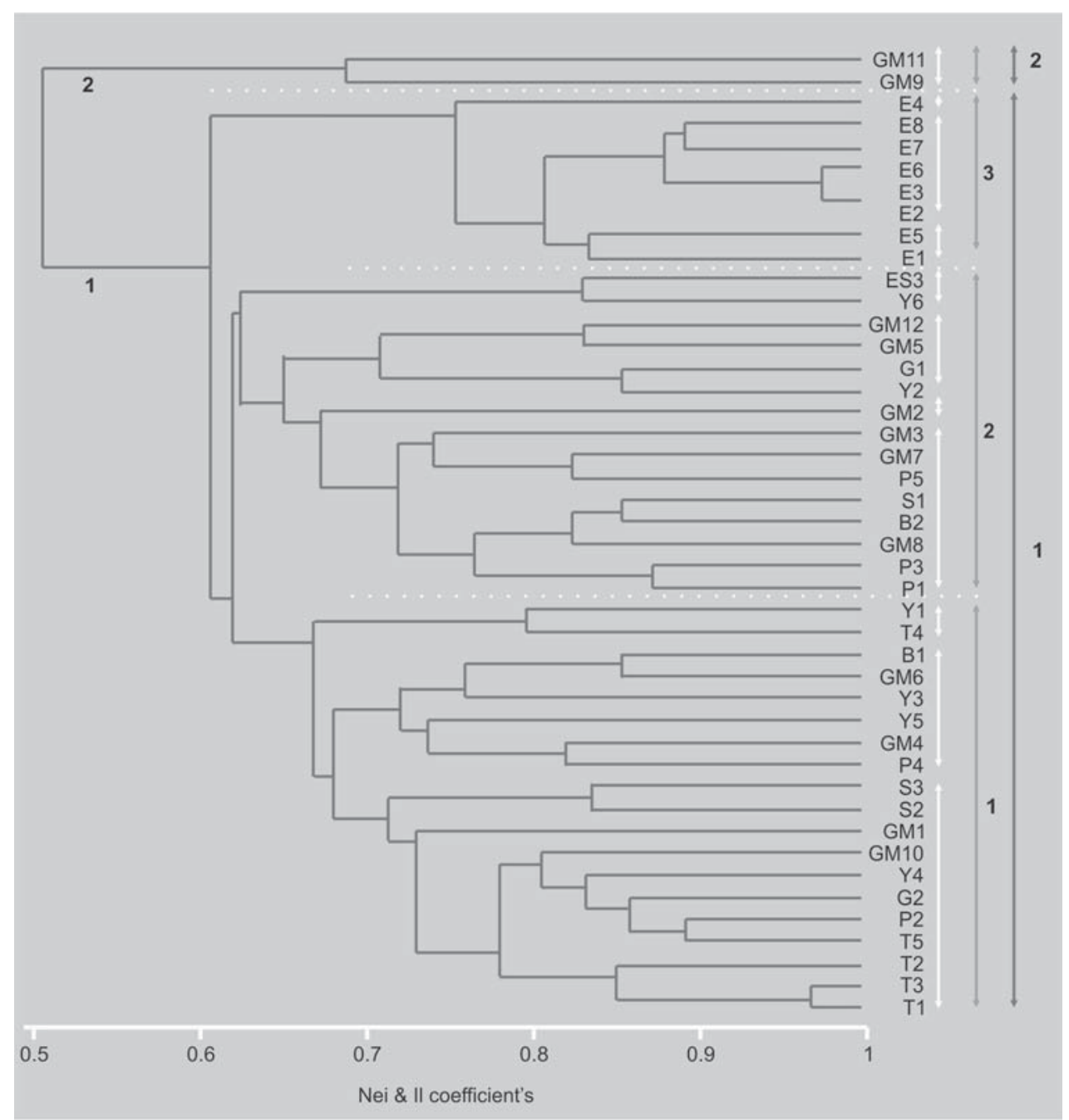

Kaçar et al. (4 alleles) [4]. The amplification of microsatellite loci in sweet cherry was possible with peach microsatellites but SSR primers that were isolated from sour cherry (PceGA77, PceGA34 and PceGA25) were uninformative for sweet cherry cultivars. This could be due to the different position of sour cherry in relation to sweet cherry in Prunus phylogeny.

The similarity index among the accessions and their dendrogram was constructed with respect to the 33 markers obtained from the use of the four primer pairs Pchpgms3, PMS2, PMS3 and PMS67 (table III). This study showed that all the investigated accessions (except 'E2' and 'E3') were clearly distinct from one other by using these four SSR primer pairs. 'E2' and 'E3' genotypes yielded very similar patterns, suggesting either they are identical genotypes or we did not have enough markers to detect variation between these genotypes. All the sweet cherry genotypes obtained from Giresun, Turkey, showed a high genetic variability (figure 2).

The dendrogram was divided into two major groups with a similarity of 0.51 (figure 2). Most of the sweet cherry genotypes were placed into the first group while only two genotypes ('GM9' and 'GM11') were placed into the second group. The 'GM9' and 'GM11' sweet cherry genotypes showed distant genetic relatedness to the other sweet cherry genotypes investigated in our 


\begin{tabular}{|c|c|c|c|c|c|}
\hline Primer pair & Expected fragments & $\begin{array}{l}\text { Expected fragment } \\
\text { size range }\end{array}$ & Reference & Obtained fragments & Obtained fragment sizes (bp) \\
\hline \multirow[t]{6}{*}{ Pchpgms3 } & 1 & 179 & [20] & 7 & \multirow{6}{*}{$\begin{array}{c}164,176,180 \\
186^{\mathrm{a}}, 198,202 \\
216\end{array}$} \\
\hline & 15 & $170-230$ & [17] & & \\
\hline & 5 & $174-189$ & [21] & & \\
\hline & 5 & $174-189$ & [2] & & \\
\hline & 4 & $170-185$ & {$[4]$} & & \\
\hline & 5 & $174-189$ & [5] & & \\
\hline \multirow[t]{3}{*}{ PMS2 } & 8 & $132-152$ & [21] & 11 & \multirow{3}{*}{$\begin{array}{c}118,122,128 \\
130,134,142 \\
144,146,148 \\
168,180\end{array}$} \\
\hline & 5 & $132-148$ & [2] & & \\
\hline & 3 & $140-148$ & {$[4]$} & & \\
\hline \multirow[t]{4}{*}{ PMS3 } & 16 & $152-200$ & [21] & 8 & $172,176,180$ \\
\hline & 11 & $147-210$ & [2] & & \multirow{3}{*}{$\begin{array}{c}184,188,192 \\
200,206\end{array}$} \\
\hline & 4 & $185-210$ & [4] & & \\
\hline & 17 & $147-200$ & [5] & & \\
\hline \multirow[t]{3}{*}{ PMS67 } & 13 & $144-191$ & [21] & 7 & \multirow{3}{*}{$\begin{array}{c}144,146,150 \\
152,156,162,165\end{array}$} \\
\hline & 3 & $150-167$ & [2] & & \\
\hline & 3 & $150-167$ & {$[4]$} & & \\
\hline
\end{tabular}

study; thus, they were separated from each other with a similarity value of 0.69 .

The other investigated genotypes placed in the first group were divided into two groups with a similarity of 0.62 .

The "E1", "E2", 'E3', 'E4', 'E5', 'E6', 'E7' and 'E8' accessions (obtained from Eynesil County) were restricted to the second cluster. The highest similarity value was determined as 0.98 between the 'E2' (possibly synonymous with 'E3') and 'E6' genotypes, and the lowest similarity value in this cluster was 0.76 ('E4').

The 'Y6' and 'ES3' genotypes were distributed among the remaining sweet cherries with a similarity of 0.63. The resemblance value of these two genotypes was determined to be 0.83 .

The genotypes in the first group were divided into two subgroups with a similarity value of 0.63. Sixteen genotypes ('T1', 'T3', 'T2', 'T5', 'P2', 'G2', 'Y4', 'GM10', 'GM1', 'S2', 'S3', 'P4', 'GM4', 'B1', 'T4' and 'Y1') were placed into the first subgroup, while fifteen genotypes ('P1', 'P3', 'GM8', 'B2', 'S1', 'P5',
'GM7', 'GM3', 'GM2', 'Y2', 'G1', 'GM5', 'GM12', 'Y6' and 'ES3') were placed into the second subgroup.

In the first subgroup, the highest similarity value determined was between the 'T1' and 'T3' genotypes (0.92), while the lowest similarity value was 0.67 . In the second subgroup, the highest and the lowest similarity values were 0.88 (between 'P1' and 'P3') and 0.63 ('Y6', 'ES3' and the other genotypes in the second subgroup), respectively.

Some morphological and phenological properties are reported (table IV). In most cases, the clusters were not in agreement with the morphological and phenological properties (table IV, figure 2).

In the 44 sweet cherry genotypes investigated, similarity ratios ranged from 0.32 (between 'E2' and 'GM11') to 0.98 (between 'E6' to 'E2' and 'E3'), with a mean value of 0.64. The results acquired show that Turkey has a rich source of genetic variability in terms of sweet cherry, confirming the results of previous studies [1-6]. In conclusion, our results demonstrate a high level of 
Table IV.

Morphological and phenological properties of 44 sweet cherry accessions from Giresun, Turkey.

\begin{tabular}{|c|c|c|c|c|c|c|c|c|c|c|}
\hline \multirow[t]{2}{*}{ Accession code } & \multicolumn{2}{|c|}{ Leaf } & \multirow{2}{*}{$\begin{array}{l}\text { Petiole length } \\
\text { (cm) }\end{array}$} & \multirow{2}{*}{\multicolumn{2}{|c|}{ Gland shape and no. }} & \multicolumn{3}{|c|}{ Leaf serratedness } & \multicolumn{2}{|c|}{ Defoliation } \\
\hline & $\begin{array}{l}\text { Length } \\
\text { (cm) }\end{array}$ & $\begin{array}{l}\text { Width } \\
\text { (cm) }\end{array}$ & & & & Shape & Density & Depth & $50 \%$ & $100 \%$ \\
\hline T1 & $11.45 \pm 2.04$ & $6.14 \pm 0.76$ & $3.69 \pm 0.69$ & Globular & 2 & Blunt & Dense & Short & 24 Nov. & 13 Dec. \\
\hline T2 & $13.48 \pm 1.76$ & $7.02 \pm 1.03$ & $4.24 \pm 0.79$ & Globular & $0-2-3$ & Blunt & Dense & Medium & 13 Dec. & 17 Dec. \\
\hline T3 & $10.93 \pm 1.21$ & $5.33 \pm 0.66$ & $2.98 \pm 0.46$ & Globular & 2 & Blunt & Dense & Short & 24 Nov. & $15 \mathrm{Dec}$. \\
\hline T4 & $12.25 \pm 1.31$ & $5.78 \pm 0.55$ & $4.11 \pm 0.51$ & Globular & 2 & Cusp & Medium & Short & 05 Nov. & 30 Nov. \\
\hline T5 & $12.60 \pm 2.53$ & $6.15 \pm 0.88$ & $3.83 \pm 0.46$ & Globular & $3-4$ & Cusp & Dense & Short & 24 Nov. & $18 \mathrm{Dec}$. \\
\hline P1 & $14.00 \pm 1.72$ & $7.43 \pm 1.16$ & $3.81 \pm 0.78$ & Globular & 2 & Blunt & Medium & Medium & 15 Dec. & $21 \mathrm{Dec}$. \\
\hline P2 & $12.83 \pm 1.43$ & $7.81 \pm 1.12$ & $4.23 \pm 0.57$ & Globular & $2-3$ & Blunt & Medium & Medium & 10 Nov. & $03 \mathrm{Dec}$. \\
\hline P3 & $13.56 \pm 1.56$ & $7.11 \pm 0.72$ & $3.88 \pm 0.62$ & Globular & $0-1-2$ & Blunt & Medium & Short & 24 Nov. & 15 Dec. \\
\hline P4 & $13.66 \pm 1.98$ & $6.94 \pm 1.15$ & $3.68 \pm 0.95$ & Reniform & 2 & Blunt & Medium & Long & 30 Nov. & $15 \mathrm{Dec}$. \\
\hline P5 & $10.14 \pm 1.98$ & $5.34 \pm 0.77$ & $4.10 \pm 0.70$ & Globular & 2 & Blunt & Dense & Medium & 06 Nov. & 02 Dec. \\
\hline Y1 & $11.53 \pm 1.58$ & $6.56 \pm 1.06$ & $3.45 \pm 0.95$ & Globular & 2 & Cusp & Dense & Long & 09 Nov. & 30 Nov. \\
\hline Y2 & $11.86 \pm 2.25$ & $6.22 \pm 1.20$ & $4.51 \pm 0.75$ & Globular & $2-3$ & Cusp & Medium & Long & 18 Nov. & 25 Nov. \\
\hline Y3 & $11.67 \pm 1.53$ & $6.83 \pm 0.91$ & $3.01 \pm 0.59$ & Globular & 2 & Cusp & Dense & Medium & 27 Nov. & $11 \mathrm{Dec}$. \\
\hline Y4 & $13.22 \pm 1.67$ & $6.19 \pm 0.51$ & $3.81 \pm 0.72$ & Globular & 2 & Cusp & Dense & Medium & $13 \mathrm{Dec}$. & 16 Dec. \\
\hline Y5 & $12.64 \pm 1.86$ & $6.84 \pm 0.72$ & $4.18 \pm 0.71$ & Globular & $0-1$ & Cusp & Dense & Medium & $01 \mathrm{Dec}$. & $15 \mathrm{Dec}$. \\
\hline Y6 & $12.83 \pm 1.93$ & $6.39 \pm 0.98$ & $4.57 \pm 0.71$ & Reniform & 2 & Cusp & Medium & Long & 12 Dec. & $21 \mathrm{Dec}$. \\
\hline ES3 & $14.05 \pm 2.61$ & $6.95 \pm 1.14$ & $3.84 \pm 0.73$ & Globular & 2 & Cusp & Dense & Medium & 07 Nov. & $17 \mathrm{Dec}$. \\
\hline GM1 & $12.00 \pm 1.31$ & $6.61 \pm 1.05$ & $4.46 \pm 0.72$ & Reniform & $1-2-3$ & Blunt & Dense & Medium & 14 Nov. & 02 Dec. \\
\hline GM2 & $13.97 \pm 0.87$ & $6.87 \pm 0.70$ & $4.81 \pm 0.48$ & Globular & $2-3-4$ & Blunt & Medium & Long & 16 Nov. & 04 Feb. \\
\hline GM3 & $11.86 \pm 1.95$ & $6.72 \pm 0.93$ & $3.84 \pm 0.69$ & Globular & $1-2$ & Cusp & Sparse & Long & 15 Nov. & 09 Dec. \\
\hline GM4 & $13.08 \pm 1.08$ & $7.00 \pm 1.08$ & $4.74 \pm 0.55$ & Globular & 2 & Cusp & Medium & Medium & 03 Dec. & $18 \mathrm{Dec}$. \\
\hline GM5 & $12.23 \pm 1.90$ & $6.81 \pm 0.80$ & $3.99 \pm 0.61$ & Reniform & 2 & Blunt & Dense & Short & $05 \mathrm{Dec}$. & 16 Dec. \\
\hline GM6 & $12.86 \pm 1.34$ & $6.43 \pm 1.37$ & $3.70 \pm 0.55$ & Reniform & $2-4$ & Cusp & Dense & Long & $13 \mathrm{Dec}$. & $16 \mathrm{Dec}$. \\
\hline GM7 & $12.61 \pm 2.25$ & $6.43 \pm 0.80$ & $4.05 \pm 0.58$ & Reniform & 2 & Cusp & Medium & Medium & 09 Nov. & $15 \mathrm{Dec}$. \\
\hline GM8 & $13.44 \pm 2.12$ & $7.14 \pm 1.14$ & $5.11 \pm 0.97$ & Globular & $2-4$ & Blunt & Dense & Short & 03 Nov. & 02 Dec. \\
\hline GM9 & $12.62 \pm 1.83$ & $6.90 \pm 0.85$ & $3.48 \pm 0.30$ & Reniform & 2 & Blunt & Dense & Medium & $13 \mathrm{Dec}$. & $18 \mathrm{Dec}$. \\
\hline GM10 & $12.86 \pm 1.67$ & $6.52 \pm 0.69$ & $3.70 \pm 0.40$ & Globular & $1-2$ & Cusp & Dense & Medium & 05 Dec. & $15 \mathrm{Dec}$. \\
\hline GM11 & $13.09 \pm 1.66$ & $7.43 \pm 0.81$ & $3.84 \pm 0.48$ & Globular & $0-1-2-3$ & Cusp & Dense & Medium & 24 Nov. & $15 \mathrm{Dec}$. \\
\hline GM12 & $11.71 \pm 1.76$ & $6.44 \pm 1.02$ & $3.78 \pm 0.50$ & Globular & 2 & Blunt & Dense & Short & 26 Nov. & $17 \mathrm{Dec}$. \\
\hline B1 & $12.63 \pm 1.52$ & $7.21 \pm 0.68$ & $3.58 \pm 0.64$ & Globular & $0-2$ & Cusp & Dense & Short & 02 Dec. & $18 \mathrm{Dec}$. \\
\hline B2 & - & - & - & - & - & - & - & - & - & - \\
\hline G1 & $10.95 \pm 1.82$ & $6.07 \pm 0.93$ & $3.27 \pm 0.52$ & Reniform & 2 & Cusp & Dense & Medium & 05 Nov. & $07 \mathrm{Dec}$. \\
\hline G2 & $12.46 \pm 1.55$ & $6.86 \pm 0.84$ & $3.25 \pm 0.75$ & Globular & $0-1$ & Blunt & Medium & Medium & 19 Nov. & $15 \mathrm{Dec}$. \\
\hline S1 & $13.87 \pm 0.69$ & $7.87 \pm 0.86$ & $3.76 \pm 0.75$ & Reniform & $2-3$ & Cusp & Dense & Short & $05 \mathrm{Dec}$. & $20 \mathrm{Dec}$. \\
\hline S2 & $11.92 \pm 2.34$ & $6.26 \pm 0.63$ & $4.41 \pm 0.75$ & Reniform & $0-2$ & Cusp & Dense & Short & 16 Nov. & $15 \mathrm{Dec}$. \\
\hline S3 & $14.55 \pm 2.09$ & $6.22 \pm 0.96$ & $4.83 \pm 0.96$ & Reniform & 2 & Blunt & Dense & Medium & 10 Nov. & $15 \mathrm{Dec}$. \\
\hline E1 & $14.76 \pm 2.44$ & $7.08 \pm 1.02$ & $3.53 \pm 0.54$ & Globular & 4 & Blunt & Dense & Short & 07 Nov. & $10 \mathrm{Dec}$. \\
\hline E2 & $13.16 \pm 1.84$ & $7.56 \pm 1.16$ & $3.86 \pm 0.54$ & Globular & $1-2-3$ & Blunt & Dense & Medium & 08 Nov. & $18 \mathrm{Dec}$. \\
\hline E3 & - & - & - & - & - & - & - & - & - & - \\
\hline E4 & $12.98 \pm 1.72$ & $6.72 \pm 0.57$ & $5.30 \pm 0.90$ & Globular & 2 & Cusp & Medium & Long & $12 \mathrm{Dec}$. & $15 \mathrm{Dec}$. \\
\hline E5 & $11.72 \pm 2.33$ & $5.76 \pm 1.34$ & $2.81 \pm 0.70$ & Globular & 2 & Blunt & Dense & Medium & 24 Nov. & $17 \mathrm{Dec}$. \\
\hline E6 & - & - & - & - & - & - & - & - & - & - \\
\hline E7 & $12.99 \pm 2.23$ & $7.54 \pm 1.02$ & $3.22 \pm 0.69$ & Globular & $0-2$ & Blunt & Medium & Short & 30 Nov. & $15 \mathrm{Dec}$. \\
\hline E8 & $13.40 \pm 2.00$ & $6.47 \pm 0.90$ & $4.63 \pm 0.63$ & Globular & $2-3$ & Blunt & Dense & Short & 18 Nov. & $16 \mathrm{Dec}$. \\
\hline
\end{tabular}


polymorphism among sweet cherry genotypes from a single province.

The findings of this research will contribute to preservation of natural resources and to breeding studies in the future. The identified and preserved genotypes will be used to select new cherry cultivars.

\section{Acknowledgements}

We gratefully acknowledge the TUBITAK, the Scientific and Technological Research Council of Turkey (Project no. TOVAG-104 O 113), for funding this project. We would also like to thank the Alara Company (Bayramiç-Çanakkale, Turkey) for supplying the sampling production of the investigated sweet cherry genotypes.

\section{References}

[1] Özbek S., Special fruit growing, Ankara Univ. Press., Ankara, Turkey, 1978.

[2] Kaçar Y.A., Classification of important sweet cherry (Prunus avium L.) and sour cherry (Prunus cerasus L.) cultivars and types growing in turkey by DNA fingerprinting methods, Çukurova Univ., thesis, Adana, Turkey, 2001, $190 \mathrm{p}$.

[3] Demirsoy L., Demirsoy H., Characteristics of some local and standard sweet cherry cultivars grown in Turkey, J. Am. Pom. Soc. 57 (2003) 128-136.

[4] Kaçar Y.A., lezzoni A.F., Çetiner M.S., Sweet cherry cultivar identification by using SSR markers, J. Biol. Sci. 5 (2005) 616-619.

[5] Kaçar Y.A., Çetiner M.S., Cantini C., lezzoni A.F., Simple sequence repeat (SSR) markers differentiate Turkish sour cherry germplasm, J. Am. Pomol. Soc. 60 (2006) 136-143.

[6] Öz M.H., Aygün H., Soydam S., Çukadar K., Bakır M., Ünlü H.M., Karadoğan B., Vurgun H., Ergül A., Molecular analysis of plum and sweet cherry genetic resources in east Anatolia by SSR, V. Natl. Hortic. Congr., Erzurum, Turkey, 2006, poster.

[7] Browicz K., Prunus, in: Davis P.H. (Ed.), Flora of Turkey and East Aegean Island, Vol. 4, Edinb. Univ. Press, Edinb., U.K., 1972, 8-12.
[8] Beaver J.A., lezzoni A.F., Ramm C.W., Isozyme diversity in sour, sweet and ground cherry, Theor. Appl. Genet. 90 (1995) 847-852.

[9] Boskovic R., Tobbut K.R., Nicoll F.J., Inheritance of isoenzymes and their linkage relationships in two interspecific cherry progenies, Euphytica 93 (1997) 129-143.

[10] Zhou L., Kappel F., Wiersma P.A., Hampson C., Bakkeren G., Genetic analysis and DNA fingerprinting of sweet cherry cultivars and selections using Amplified Fragment Length Polymorphisms (AFLP), Acta Hortic. 667 (2005) 37-44.

[11] Williams J.G.K., Kubelik A.R., Livak K.J., Rafalski J.A., Tingey S.V., DNA polymorphisms amplified by arbitrary primers are useful as genetic markers, Nucleic Acid Res. 18 (1990) 6531-6535.

[12] Yang H., Schmitt H., Selection of a mutant from adventitious shoots formed in X-ray treated leaves and differentiation of standard from mutant with RAPDs, in: Schmith $\mathrm{H}$., Kellerhals M. (Eds.), Progress in temperate fruit breeding, Kluwer Acad. Publ., Dordrecht, Neth., 1994, pp. 287-290.

[13] Corona M.G., Resta P., Fanizza G., Palasciano M., Godini A., Prima indagine su cultivar pugliesi di ciliegio dolce (Prunus avium L.) mediante lo studio di polimorfismi del DNA (RAPDs), in: Godini A. (Eds.), Atti del Covegno Nazionale del Ciliegio, Valezano (Ba), Italy, 1997, pp. 201-213.

[14] Gerlach H.K., Stösser R., Sweet cherry cultivar identification using RAPD derived DNA fingerprints, Acta Hortic. 468 (1998) 63-70.

[15] Demirsoy L., Demir T., Demirsoy H., Kaçar Y.A., Okumuş A., Identification of some sweet cherry cultivars grown in Amasya by RAPD markers, Acta Hortic. 795 (2008) 147-153.

[16] Boritzki M., Plieske J., Struss D., Cultivar identification in sweet cherry (Prunus avium L.) using AFLP and microsatellite markers, Acta Hortic. 538 (2000) 505-510.

[17] Downey S.L., lezzoni A.F., Polymorphic DNA markers in black cherry (Prunus serotina) are identified using sequences from sweet cherry, peach and sour cherry, J. Am. Soc. Hortic. Sci. 125 (2000) 76-80.

[18] Struss D., Ahmad R., Southwick S.M., Boritzki M., Analysis of sweet cherry (Prunus avium L.) cultivars using SSR and AFLP 
markers, J. Am. Soc. Hortic. Sci. 128 (2003) 904-909.

[19] Tavaud M., Zanetto A., Santi F., Dirlewanger E., Structuration of genetic diversity in cultivated and wild cherry varieties using molecular markers, Acta Hortic. 546 (2001) 263-269.

[20] Sosinski B., Gannavarapu M., Hager L.D., Beck L.E., King G.J., Ryder C.D., Rajapakse S., Baird W.V., Ballard R.E., Abott A.G., Characterization of microsatellite markers in peach, Theor. Appl. Genet. 97 (2000) 264-272.

[21] Cantini C., lezzoni A.F., Lamboy W., Boritzki M., Struss D., DNA fingerprinting of tetraploid cherry germplasm using simple sequence repeats, J. Am. Soc. Hortic. Sci. 126 (2001) 205-209.

[22] Nei M., Li W., Mathematical model for studying genetic variance in terms of restriction endonucleases, Proc. Natl. Acad. Sci. 76

\section{Caracterización molecular de los recursos genéticos del cerezo en la provincia de Giresun en Turquía.}

Resumen - Introducción. Turquía sería potencialmente una fuente muy rica de cerezos dulce (Prunus avium) y ácido (Prunus cerasus). La especie P. avium supuestamente es originaria de ciertas regiones del norte de Turquía, entre las cuales se halla la provincia de Giresun. La identificación de los cultivares de cerezos turcos debería ayudar a poder elegir y a contribuir en la conservación de los recursos naturales necesaria para trabajos de mejora. El método más convencional para la identificación de cultivares está basado en la evaluación de las características morfológicas. No obstante, dicho método es insuficiente para distinguir los cultivares estrechamente relacionados. Los objetivos de nuestro estudio pretendieron determinar el perfil molecular de muestras de cerezos producidos en la provincia de Giresun en Turquía, así como determinar sus distancias genéticas. Material y métodos. Nuestro estudio permitió identificar 44 muestras de cerezos gracias al empleo de marcadores genéticos, así como determinar sus distancias genéticas. Aislamos el ADN de las jóvenes hojas extraídas de un plantón de muestra, y efectuamos la amplificación de los loci de microsatélites. En total, se emplearon diez pares de cebador de SSR, previamente aislados de melocotonero y de cerezo. Se calcularon índices de similitud. Se utilizó un análisis tipológico para trazar un dendograma. Resultados y discusión. De los diez cebadores examinados, seis no permitieron obtener productos de amplificación a partir de las 44 muestras estudiadas. Los cuatro pares de cebadores polimorfos restantes produjeron 33 alelos con una media de 8.25 alelos putativos por locus. Según las muestras, los índices de similitud variaron entre 0.32 y 0.98 , con un valor medio de 0.64. En conclusión, los resultados obtenidos revelan un elevado nivel de polimorfismo entre los genotipos de cerezo estudiados en una sola provincia de Turquía.

Turquía / Prunus avium / recursos genéticos / identificación / microsatélites / marcadores genéticos / distancia genética 